\title{
Scale dependence of branching in arterial and bronchial trees
}

\author{
Juan G. Restrepo, ${ }^{1,2, \text { : Edward Ott, },^{1,3} \text { and Brian R. Hunt }}{ }^{2,4}$ \\ 1 Institute for Research in Electronics and Applied Physics, \\ University of Maryland, College Park, Maryland 20742, USA \\ 2 Department of Mathematics, University of Maryland, College Park, Maryland 20742, USA \\ ${ }^{3}$ Department of Physics and Department of Electrical and Computer Engineering, \\ University of Maryland, College Park, Maryland 20742, USA \\ 4 Institute for Physical Science and Technology, University of Maryland, College Park, Maryland 20742, USA
}

(Dated: November 20, 2018)

\begin{abstract}
Although models of branching in arterial and bronchial trees often predict a dependence of bifurcation parameters on the scale of the bifurcating vessels, direct verifications of this dependence by comparison with data are uncommon. We compare measurements of bifurcation parameters of airways and arterial trees of different mammals as a function of scale to general features predicted by theoretical models based on minimization of pumping power and network volume. We find that the size dependence is more complex than existing theories based solely on energy and volume minimization, and suggest additional factors that may govern the branching at different scales.
\end{abstract}

The potential factors determining the parameters at bifurcations in the arterial and bronchial trees of mammals have lately received attention. Most notably, these local bifurcation characteristics have been proposed to determine allometric scaling laws for biologically important variables (see [1, 2] and [3]). It has been suggested that arterial and bronchial trees follow a pattern that minimizes a combination of pumping power and the total volume of the tree (see for example [1]-[ $\underline{6}]$ ). If the flow is assumed to be Poiseuille (resistance proportional to the inverse fourth power of the vessel radius) on all scales, self-similar branching results, i.e., the branching parameters do not depend on the scale. However, it has been argued 1 that, due to the fact that the blood flow is pulsatile [8, 9], arterial trees determined by optimization have branching parameters that depend on the scale (the form of this dependence is discussed below). As a direct test of these theories and their assumptions, we compare in this Letter experimental measurements of the scale dependence of the branching parameters with the predictions of theoretical models. Many studies of bifurcation parameters rely on ordering schemes [10] which do not directly consider the dependence of the bifurcation parameters on the scale, and thus are not appropriate for our particular purpose. We will explicitly study the dependence of the radii of the bifurcated vessels on the radius of the parent vessel, thus allowing a more direct comparison with theoretical models. We show that important aspects of previous observations [11] for the human bronchial tree, not predicted by the theoretical models, are more general than has been previously noticed. In particular, we find qualitatively similar behavior for dog arterial and bronchial trees, and lamb fetal and neonatal arterial trees. Our results suggest that, in addition to minimizing pumping power and network volume, there might be other important factors determining the

*Electronic address: juanga@math.umd.edu branching in the arterial and bronchial trees.

Arterial and bronchial trees start with a tree root (a parent vessel) that divides into two (daughter) vessels, and these in turn subdivide in a similar fashion, continuing until a certain approximate value of the radius is attained (in the case of arterial trees, the capillary radius). At a particular bifurcation, we let $r_{0}$ denote the radius of the parent vessel and $r_{1}, r_{2}$ denote the radii of the daughter vessels. Variables often used to characterize the bifurcations are the area ratio and the symmetry index, defined respectively by $a=\left(r_{1}^{2}+r_{2}^{2}\right) / r_{0}^{2}$ and $\alpha=r_{2} / r_{1}$, where $r_{2} \leq r_{1}[12$. These two variables completely determine $r_{1}$ and $r_{2}$ in terms of $r_{0}$. The branching exponent $x$ defined for $r_{0} \geq r_{1} \geq r_{2}$ by $r_{0}^{x}=r_{1}^{x}+r_{2}^{x}$ is also often used in modeling and theoretical discussions [13][19]. Most predictions of theoretical models are stated in terms of this exponent. However, we find this exponent inconvenient for our purposes since it diverges as $r_{1}$ or $r_{2}$ approach $r_{0}$, as occurs frequently with experimental data. We will therefore use the area ratio in our analysis. The value of the exponent $x$ can be linked to the change of certain quantities at bifurcations. An exponent $x=2$ indicates conservation of total cross sectional area $(a=1)$ and a value $x=3$ corresponds to conservation of laminar shear stress at a bifurcation. The area ratio $a$ is related to $x$ and the symmetry index $\alpha$ by $a=\left(1+\alpha^{2}\right)\left(1+\alpha^{x}\right)^{-\frac{2}{x}}$. This is a monotonic function of $x$ which does not change much as the value of $\alpha$ is varied between 0.5 and 1 . We will therefore regard the general features of the area ratio as a function of the scale as a counterpart of a similar qualitative behavior of the exponent $x$.

Murray [4] proposed that the arterial tree is constructed in such a way as to minimize a linear combination of the pumping power and the volume of the network. This leads, if Poiseuille flow is assumed throughout the tree, to Murray's Law, which states that $x=3$. Murray's Law has been useful as a reference point regarding the small blood vessels and is sometimes mentioned in discussions regarding the bronchial tree as well [20]. However, deviations have been apparent for some time 
(see [16]-23] and references therein). The exponent $x$ has been observed to be generally smaller than the proposed value of 3 .

Recently, West et al. [1] included the effect of the blood flow being pulsatile, and found that the minimization scheme carried on by Murray leads in this case to a transition scenario, with $x$ close to 2 for the larger vessels and to 3 for the smaller vessels (see, however, Ref. [3]). This modification predicts a smooth transition from the larger to the smaller vessels, as suggested also by Uylings [5] on other grounds. Other modifications have been proposed predicting an exponent between 2 and 3 for the smaller vessels [6, 7]. In order to test these theories we study data for the bifurcation parameter $a$ as a function of the parent vessel radius. Zamir 12 presents data for the branching parameters as a function of the radius for the right coronary artery of a human heart. The large scatter in the data, however, makes it difficult to observe the dependence of the mean values on the scale. Phillips and Kaye [11] studied the dependence of averaged branching parameters on the radius of the vessels in the bronchial tree of four mammalian species. We show that some important aspects of their observations for the human bronchial tree (to be discussed) are more general than has been previously noticed.

Data sets. We study morphological data from the bronchial tree of a human and 2 dogs obtained by Raabe et al. 24], a human bronchial tree from Horsfield et al. [25], dog lung arterial trees from Dawson et al. 19] and neonatal and fetal lamb lung arterial trees from Bennett et al. 18] (see Table 1). For each data set, we compute the average value for the area ratio $\langle a\rangle$ as a function of the parent vessel radius $r$ as follows. For a given parent vessel radius $r$ we construct a sample of all bifurcations $\left(r_{0}, r_{1}, r_{2}\right)$ which satisfy $r 10^{-\epsilon}<r_{0}<r 10^{\epsilon}$, thus making uniform intervals in $\log r$, and take $\langle a\rangle$ evaluated at $r$ to be the average of $a$ over this finite sample. We choose the logarithmic interval width $2 \epsilon$ to be 0.05 . This value was chosen so that the statistical noise is filtered but the large scale features of $\langle a\rangle$ as a function of $r$ are not smoothed out. For the largest vessels and sometimes for the very small vessels, the number of bifurcations measured is sparse, especially when only one lung was measured. We reject the values obtained when the sample for a given range of radii contains less than 4 members.

\begin{tabular}{|r|r|r|r|r|r|}
\hline Data set & Mammal & Tree & Body mass (kg) & Radius range (cm) & Bifurcations \\
\hline Horsfield et al. & Human 1 & Bronchial & - & $0.07-1.6$ & 3088 \\
\hline Raabe et al. & Human 2 & Bronchial & 81 & $0.04-2.01$ & 4336 \\
\hline Raabe et al. & Dog 1 & Bronchial & 11.6 & $0.02-1.8$ & 8032 \\
\hline Raabe et al. & Dog 2 & Bronchial & 10.3 & $0.03-1.61$ & 2057 \\
\hline Dawson et al. & Dogs (32) & Lung Arterial & $19.7 \pm 2.1$ & $0.003-0.76$ & 919 \\
\hline Bennett et al. & Lamb (fetal) & Lung Arterial & - & $0.001-0.70$ & 10970 \\
\hline Bennett et al. & Lamb (neonatal) & Lung Arterial & - & $0.011-1.23$ & 846 \\
\hline
\end{tabular}

TABLE I: Characteristics of the different data sets used. A "-" in the body mass entry indicates that this information was not available

The results of this procedure are shown in Figure 1] In order to allow comparisons with the values of the exponent $x$ predicted by theoretical models, we display the values of $a$ corresponding to $x=3$ as a thick line on the right axis. The corresponding value of the area ratio $a$ for $x=3$ depends on the symmetry index $\alpha$, and thus we marked the range of values of $a$ corresponding to $x=3$ for values of $\alpha$ ranging from 0.5 to 1 . The error bars correspond to one standard deviation as computed from the propagated measurement error taken from the precision to which the data were reported, and the statistical uncertainty due to a finite sample.

In Figs. 1(a) and (b) we show $\langle a\rangle$ as a function of the parent radius $r$ for the human and $\operatorname{dog}$ bronchial trees respectively, obtained as described above from the Raabe et al. and Horsfield et al. data sets. In Fig. 1(c) we display the area ratio from the dog pulmonary arterial tree, and fetal and neonatal lamb arterial trees from the data of Dawson et al. and Bennett et al., respectively. The behavior for the largest vessels is not shown in the plots because of lack of good statistics. It has been shown elsewhere [27] that the bifurcations of the largest vessels in the arterial tree generally have an area ratio close to 1 .

We first discuss the results for the dog and human bronchial trees [Figs. 1(a) and (b)]. The behavior of $\langle a\rangle$ as a function of $r$ is not a simple one: it is not constant, as it would be if the branching were self-similar, nor does it have a smooth transition from a lower value to a higher value as $r$ decreases. Remarkably, the same qualitative behavior is observed in both graphs. This behavior consists of the following pattern: for the very small and close to terminal vessels, the area ratio is high. As $r$ increases, $\langle a\rangle$ decreases, reaching a local minimum at $r \sim 1-2 \mathrm{~mm}$. Then the area ratio grows, and, for Human 1 (boxes in [Fig. 1(a)]) and the dogs [Fig. 11(b)], one can observe it 

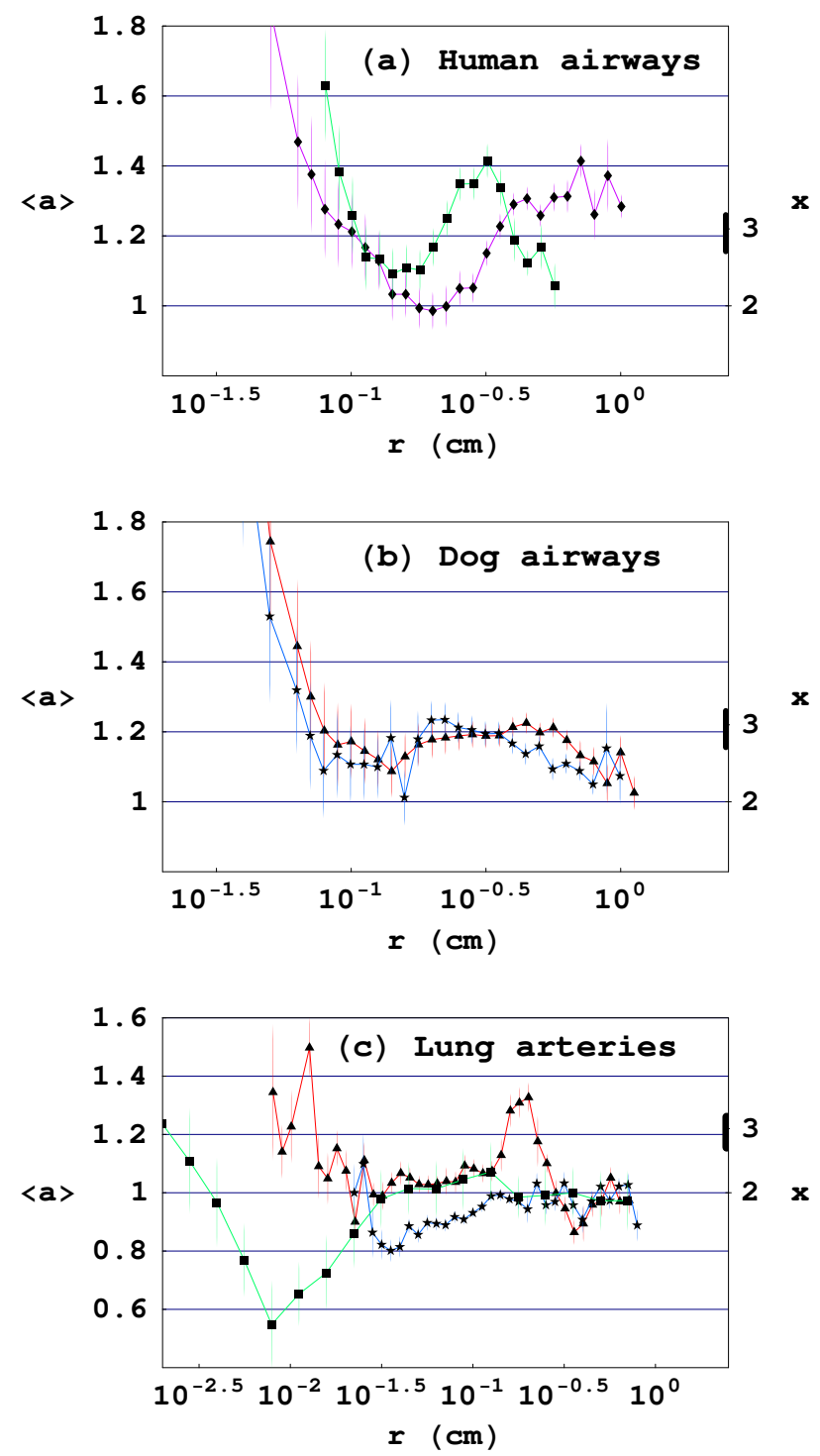

FIG. 1: Average area ratio $\langle a\rangle$ as a function of the parent airway radius $r$ for (a) Horsfield et al. Human 1 (boxes) and Raabe et al. Human 2 (diamonds), (b) Raabe et al. Dog 1 (triangles) and Dog 2 (stars) (see Table 1), and (c) Dawson et al. dogs lung arterial tree (triangles) and Bennett et al. neonatal (stars) and fetal (diamonds) lambs lung arterial tree.

finally decreasing again. In quantitative terms, we make the following observations. For the humans, among the largest vessels, the value of the area ratio $\langle a\rangle$ peaks higher than that corresponding to a value of $x=3$, even when the error bars are taken into account. For smaller vessels, $\langle a\rangle$ decreases as mentioned before, attaining values below those equivalent to $x=3$. For the smallest vessels, the area ratio takes values substantially above those corresponding to $x=3$.
We observe a qualitatively similar situation for the lung arteries [Fig. [(c)]. For each data set, there is a local minimum of the area ratio at moderately small vessel radius, and the area ratio increases sharply as the radius decreases further. The fetal and neonatal lamb arterial trees (diamonds and stars, respectively) have smaller overall values of the area ratio, as noted before [18]. In the dog arterial tree (triangles) the different regimes are particularly marked: area ratio close to 1 for the largest vessels, a peak for the moderately large vessels, a smaller value of the area ratio as the radius decreases further, and larger values of the area ratio near the terminal vessels. The presence of this peak for moderately large vessels was noted by Phillips et al. 11] for the bronchial tree of the human in Raabe et al. data set; we see that this behavior may be more general.

We also studied data for the airways of two rats and a hamster from Raabe et al. 24], but the range of vessel radii was smaller and the results were not conclusive. Data from the heart arteries of a pig from Kassab et al. 26] also did not show a clear dependence of area ratio on vessel radius. While not inconsistent with our observations above, they were not inconsistent with the hypothesis of a constant area ratio.

The above discussion is qualitative, and it is natural to ask whether it can be supported by a rigorous statistical test. We believe, however, that due to the limited amount of samples available and to the inherent differences between each of the sampled individuals, we do not have sufficient basis for formulating an alternative model to those we have discussed. We hope that the graphical information we have presented will encourage experimentalists to measure and make available more data.

In summary, a simple transition of the area ratio from values close to 1 for the large vessels to higher values for the smaller vessels, as predicted by some optimization models, is not supported by the data we studied. The assumption that an optimization principle is involved in the design of the arterial and bronchial trees is natural, as evolution would tend to eliminate designs that are not adapted to meet the demands of the organisms. We believe, however, that these demands are not restricted to energy efficiency (as assumed in most theories) and that different factors compete, resulting in the complex behavior of the area ratio. As has been remarked recently [28], a bronchial tree designed solely with the purpose of minimizing pumping power and network volume could be dangerously sensitive to deviations from the optimal bifurcation values, and higher area ratios could be a safety margin against the effect of these variations. Other factors possibly contributing to make the area ratio smaller for the largest vessels are impedance matching and minimization of the resistance for pulsatile flow 1]. A higher value of the area ratio as the radius decreases could indicate a tendency to relieve the high pressure and shear stress present in the large vessels. The large value of the area ratio near the terminal vessels could allow the fluid to be slowed down as it reaches the capillaries or alveoli 


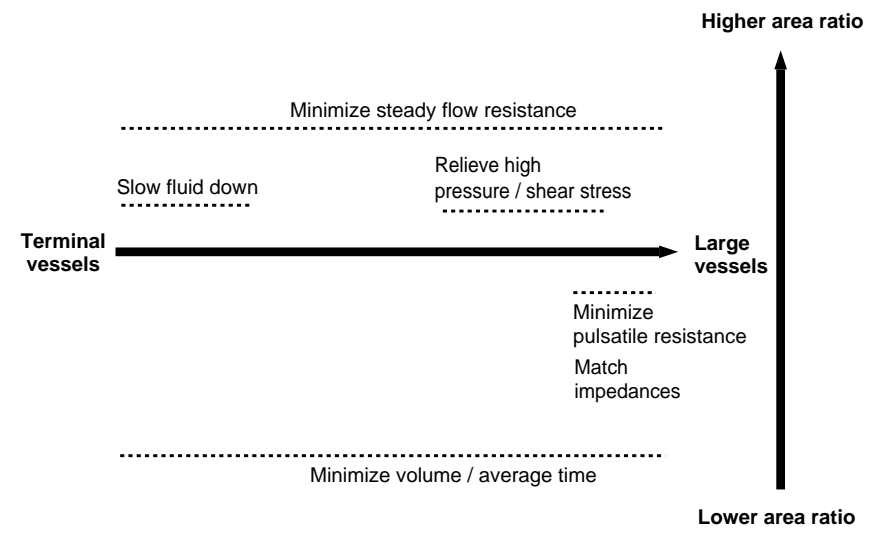

FIG. 2: Schematic representation of possible factors affecting the area ratio on different scales. The factors above the thick horizontal line could favor higher area ratios, and those below the horizontal line favor a lower area ratio (our vertical order is otherwise arbitrary). A compromise between these competing factors could produce transitions as seen in Fig. 1.

(e.g., to allow fuller transfer of the transported nutrient to the body). On the other hand, minimizing the volume or the average circulation time favors a smaller area ratio on all scales. Also, the effects of finite vessel length and vessel curvature could potentially affect the values of the optimal branching parameters. This discussion is summarized in Fig. 2 We emphasize that these are only possible factors, and that this list is not meant to be exhaustive. In conclusion, we have presented evidence of a consistent and so far unexplained behavior in diverse arterial and bronchial trees. To explain these observations it might be necessary to couple existing optimality principles to other requirements. The precise formulation and understanding of such constraints is a nontrivial and important problem. The consequences for allometric scaling are also important, as the branching pattern of the arterial tree has been argued 1] to be determinant of the scaling of many variables. We believe it will be fruitful to carry on a similar analysis on other data.

Acknowledgements: we thank C. A. Dawson and S. H. Bennett for kindly providing us with data. We also acknowledge the Lovelace Respiratory Research Institute for allowing us to use their Report LF-53.
[1] G. B. West, J. H. Brown, B. J. Enquist, Science 276, 122 (1997); G. B. West and J. H. Brown, Physics Today 57 (9), 36 (2004). The term allometric scaling refers to the scaling of average variables characterizing different species across a wide range of animals; e.g., metabolism versus body mass for land mammals ranging from the smallest size (shrew) to the largest size (elephant).

[2] O. Dreyer, Phys. Rev Lett. 87, 038101 (2001).

[3] P. S. Dodds, D. H. Rothman, and J. S. Weitz, J. Theor. Biol., 209, 9, (2001).

[4] C. D. Murray, Proc. Natl. Acad. Sci. U.S.A. 12, 207 (1926).

[5] H. B. M. Uylings, Bull. Math. Biol. 39, 509 (1977).

[6] S. Oka, M. Nakai, Biorheology 24, 737 (1987).

[7] Z. Yifang, G. Kassab, S. Molloi, Phys. Med. Biol. 44, 2929 (1999).

[8] M. Zamir, The Physics of Pulsatile Flow, (SpringerVerlag, New York, NY, 2000).

[9] W.S. Duan, B.R. Wang, and R.J. Wei, Phys. Rev. E 55, 1773 (1997).

[10] K. Horsfield, F. G. Relea, G. Cumming, Respir. Physiol. 26, 351 (1976).

[11] C. G. Phillips, S. R. Kaye, Respir. Physiol. 102, 303 (1995).

[12] M. Zamir, J. Theor. Biol. 197, 517 (1999).

[13] M. Woldenberg, K. Horsfield, J. Theor. Biol. 104, 301 (1983).

[14] R. Karch, F. Neumann, M. Neumann, W. Schreiner, Ann. Biomed. Eng. 28, 496 (2000).
[15] K. L. Karau, G. S. Krenz, C. A. Dawson, Am. J. Physiol. Heart Circ. Physiol. 280, H1256 (2001).

[16] H. Kitaoka, B. Suki, J. Appl. Physiol. 82, 968 (1997) .

[17] M. A. Changizi, C. Cherniak, Can. J. Physiol. Pharmacol. 78, 603 (2000).

[18] S. H. Bennett et al., Am. J. Physiol. Heart Circ. Physiol. 279, H3047 (2000).

[19] C. A. Dawson et al., J. Appl. Physiol. 86, 569 (1999).

[20] E. Weibel, The pathway for oxygen (Harvard University Press, Cambridge, MA, 1984).

[21] M. Pollanen, J. Theor. Biol. 159, 267 (1992) .

[22] K. Horsfield, M. J. Woldenberg, Anat. Rec. 223, 245 (1989).

[23] K. Horsfield, A. Thurlbeck, Bull. Math. Biol. 43, 681 (1981).

[24] O. G. Raabe, H. C. Yeh, H. M. Schum, R. F. Phalen, "Tracheobronchial geometry- human, dog, rat, hamster" (Report LF-53, Lovelace Foundation for Medical Education and Research, Albuquerque, NM, 1976).

[25] K. Horsfield, G. Cumming, J. Appl. Physiol. 24, 373 (1968).

[26] G. S. Kassab, C. A. Rider, N. J. Tang, Y. C. Fung, Am. J. Physiol. Heart Circ. Physiol. 265, H350 (1993).

[27] M. Zamir, P. Sinclair, T. H. Wonnacott, J. Biomech. 25, 1303 (1992).

[28] B. Mauroy, M. Filoche, E. R. Weibel, B. Sapoval, Nature 427, 633 (2004). 